\title{
Control y supervisión de un proceso electro-neumático
}

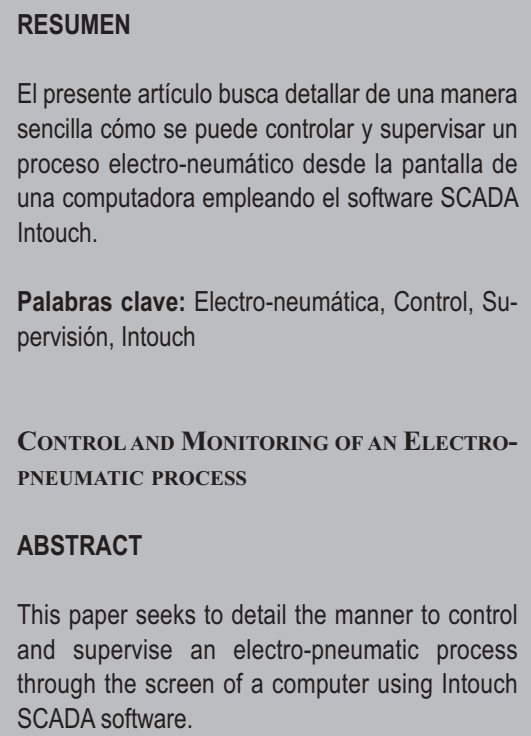

Palabras clave: Electro-neumática, Control, Supervisión, Intouch

Control and Monitoring of an ElectroPNEUMATIC PROCESS

\section{INTRODUCCIÓN}

La incorporación de herramientas de automatización en los procesos industriales es una necesidad para toda empresa que busca sobresalir en su propio mercado y en los internacionales.

La electro-neumática, los controladores lógicos programables (PLC's), la robótica, los sistemas de supervisión y control (SCADA), y en general de la automatización, concentran un conjunto de tecnologías y aplicaciones cuya implantación industrial incrementa drásticamente la productividad, permitiendo en paralelo la mejora de calidad de los productos y de las condiciones de trabajo del personal de planta.

El control de un sistema electro-neumático puede ser realizado a través de un PLC con características que permitan el óptimo desempeño de los elementos a controlar. Para este proyecto se empleará un PLC Festo de tipo compacto, al cual se conectaran 3 cilindros de doble efecto, a 3 electro-válvulas biestables, cada una de ellas con un.

La supervisión del sistema se logrará mediante el uso del software Intouch, ello permitirá que todas las variables del proceso se muestren en la pantalla de un computador. Esta es una ventaja para iniciar o detener la secuencia del sistema electro-neumático, porque solo bastará con oprimir un botón del mouse.

\section{DISEÑO DEL MODELO NEUMÁTICO Y ELECTRO-NEUMÁTICO}

El diseño del sistema se realizará en el software FluidSim, el cual también permite simular secuencias de sistemas neumáticos y electro-neumáticos.

Para iniciar, se colocara en el proyecto los siguientes dispositivos: fuente de aire comprimido, unidad de mantenimiento, pulsadores, electro-válvulas tipo $5 / 2$ biestables, finales de carrera y sensores.

En la Figura $\mathrm{N} .^{\circ} 1$ se muestra el diseño del sistema neumático propuesto para la secuencia A+B+C+A-B- C-. Esto es, al presionar un pulsador se extiende el cilindro $A$; cuando ello ocurre, se activa el final de carrera $A 1$, quien envía una señal para que el cilindro $B$ se extienda; cuando ello ocurre, se activa el final de carrera B1, quien envía una señal para que el cilindro $C$ se extienda; cuando ello ocurre, se activa el final de carrera $C 1$, quien envía una señal para que el cilindro $A$ se re-

Jefe del Laboratorio de Automatización - CIM (UNIVERSIDAD RICARDO PALMA. PERU) email: avelasquez@urp.edu.pe; cim.urp@gmail.com 
Figura N. $^{\circ}$ 1: Diseño neumático del sistema A+ B+ C+ A- B- C-



FUENTE: Elaboración propia

traiga; cuando ello ocurre, se activa el final de carrera $\mathrm{A} 0$, quien envía una señal para que el cilindro $B$ se retraiga; cuando ello ocurre, se activa el final de carrera B0, quien finalmente envía una señal para que el cilindro $C$ se retraiga. El ciclo puede ser único o continuo. Será ciclo único si se presiona el pulsador rasante, y ciclo continuo, si se presiona el pulsador con enclavamiento.

La Figura N ${ }^{\circ} 2$ muestra el diseño electro-neumático del sistema propuesto. Aquí se han asignado distinto tipos de detectores para las posiciones retraídas y extendidas de los cilindros de doble efecto. La tabla 1 muestra las señales de entrada de los finales de carrera y sensores que se conectan al PLC para permitir la supervisión del sistema.

Según el diseño propuesto, la simulación muestra concretamente la secuencia $\mathrm{A}+\mathrm{B}+\mathrm{C}+\mathrm{A}-\mathrm{B}-\mathrm{C}-$. Cada letra representa un cilindro, en este caso se tiene 3 cilindros, el símbolo "+" representa el cilindro extendido, el símbolo "-" representa el cilindro retraído. Visto de esta manera, se logra detallar lo que ocurre en el circuito electro-neumático:

Al presionar un pulsador eléctrico conmuta la válvula de control del cilindro A conmute y permite que
Figura N. 2: Diseño electro-neumático del sistema $A+B+C+A-B-C-$



FUENTE: Elaboración propia.

el aire comprimido ingrese a la válvula, llegue al cilindro y se extienda el cilindro. Una vez extendido el cilindro A, el sensor óptico A1, detecta dicha posición y envía una señal para que la válvula que controla al cilindro B conmute y permita que este

Tabla N. ${ }^{\circ}$ 1: Señales de entrada para los cilindros

\begin{tabular}{|c|c|c|}
\hline & Posición retraído & Posición extendido \\
\hline Cilindro A & Final de carrera (A0) & Sensor óptico (A1) \\
\hline Cilindro B & Final de carrera (B0) & Sensor óptico (B1) \\
\hline Cilindro C & Final de carrera (C0) & Final de carrera (C1) \\
\hline
\end{tabular}

FUENTE: Elaboración propia. 
cilindro se extienda. Cuando ello ocurre, el final de carrera B1 se activa y envía una señal para que la válvula de control del cilindro $\mathrm{C}$ también conmute y se extienda dicho cilindro. Al estar extendido, se activa el final de carrera $\mathrm{C} 1$ y permite el retroceso del cilindro A, luego de cilindro B y, finalmente, del tercer cilindro.

\section{MONTAJE DE LA APLICARON REAL}

El montaje del sistema electro-neumático propuesto se realiza sobre una placa perfilada en la cual se colocarán cada uno de los elementos del sistema electro-neumático.

Las direcciones de las señales que se conectaran al PLC se muestran en la Tabla N. ${ }^{\circ} 2$ :

Tabla N. ${ }^{\circ}$ 2: Señales de entrada y salida del PLC

\begin{tabular}{|c|c|}
\hline Dirección & Comentario \\
\hline$I 0.0$ & Pulsador de inicio \\
\hline 10.1 & Pulsador de inicio con enclavamiento \\
\hline$I 0.2$ & Final de carrera A0 \\
\hline$I 0.3$ & Sensor óptico A1 \\
\hline 10.4 & Final de carrera B0 \\
\hline 10.5 & Sensor óptico B1 \\
\hline 10.6 & Final de carrera C0 \\
\hline 10.7 & Final de carrera C1 \\
\hline 00.0 & Extender cilindro A \\
\hline 00.1 & Retraer cilindro A \\
\hline 00.2 & Extender cilindro B \\
\hline 00.3 & Retraer cilindro B \\
\hline 00.4 & Extender cilindro C \\
\hline 00.5 & Retraer cilindro C \\
\hline
\end{tabular}

FUENTE: Elaboración propia

La Figura N. ${ }^{\circ} 3$ muestra las conexiones de las entradas y salidas al PLC de la aplicación real. Se debe precisar que este PLC trabaja con 24VDC al igual que el bloque de pulsadores, es por ello que se requiere una fuente externa estabilizada que proporcione dicho voltaje.

La Figura N. ${ }^{\circ} 4$ muestra la disposición del PLC que se conectará a las ocho entradas y seis salidas correspondientes del sistema electro-neumático.

\section{Control del sistema propuesto}

El control de esta secuencia se realizará a través de un PLC compacto Festo, el cual tiene capacidad para conectar hasta un máximo de 12 entradas y 8 salidas. El programa de control será el siguiente:

Figura N.ํ 3: Aplicación real del sistema electro-neumático



Figura N..$^{\circ}$ : PLC Compacto Festo

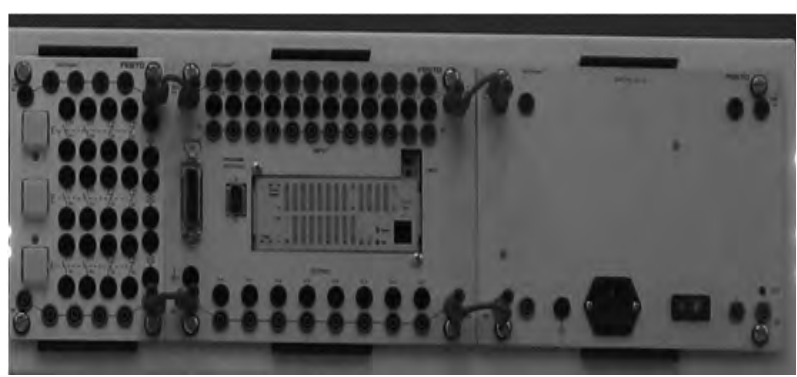

STEP 1

IF

THEN RESET

RESET O0.1

RESET O0.2

RESET O0.3

RESET $\quad 00.4$

RESET 00.5

\section{STEP 2}

IF

$\begin{array}{cc} & 10.0 \\ \text { OR } & 10.1 \\ \text { OR } & \text { F0.0 } \\ \text { OR } & \text { F0.1 }\end{array}$

THEN JMP TO 3

STEP 3

IF

AND $\quad \mathrm{N}$ T1

THEN SET

RESET

SET

WITH
00.0

00.1

T1

$3 s$
'TIMER 1

'Extender cilindro A 'Retraer cilindro A 'Extender cilindro B 'Retraer cilindro B 'Extender cilindro C 'Retraer cilindro C

'Pulsador de inicio 'Pulsador de inicio con enclavamiento 'FLAG INICIO 'FLAG INICIO CON ENCLAVAMIENTO

'Final de carrera C0 'TIMER 1

'Extender cilindro A 'Retraer cilindro A 'TIMER 1 


\begin{tabular}{|c|c|c|}
\hline \multicolumn{3}{|l|}{ STEP 4} \\
\hline IF & 10.3 & 'Sensor óptico A1 \\
\hline AND & T1 & 'TIMER 1 \\
\hline THEN SET & 00.2 & 'Extender cilindro B \\
\hline RESET & 00.3 & 'Retraer cilindro B \\
\hline SET & T1 & 'TIMER 1 \\
\hline \multicolumn{3}{|l|}{ STEP 5} \\
\hline IF & 10.5 & 'Final de carrera B1 \\
\hline AND & T1 & 'TIMER 1 \\
\hline THEN SET & 00.4 & 'Extender cilindro C \\
\hline RESET & 00.5 & 'Retraer cilindro C \\
\hline SET & T1 & 'TIMER 1 \\
\hline
\end{tabular}

STEP 6

\begin{tabular}{cc} 
IF & \multicolumn{2}{c}{10.7} \\
AND $N$ & T1 \\
THEN SET & O0.1 \\
RESET & O0.0 \\
SET & T1
\end{tabular}

\begin{tabular}{|c|c|c|}
\hline $\begin{array}{l}\text { STEP } 7 \\
\text { IF }\end{array}$ & 10.2 & 'Final de carrera $\mathrm{AO}$ \\
\hline AND & T1 & 'TIMER 1 \\
\hline THEN SET & 00.3 & 'Retraer cilindro B \\
\hline RESET & 00.2 & 'Extender cilindro B \\
\hline SE $\rceil$ & T1 & TIMER 1 \\
\hline
\end{tabular}

\begin{tabular}{|c|c|c|}
\hline $\begin{array}{l}\text { STEP } 8 \\
\text { IF }\end{array}$ & 0.4 & 'Sensor inductivo B0 \\
\hline AND & T1 & ‘TIMER 1 \\
\hline THEN SET & 00.5 & 'Retraer cilindro C \\
\hline RESET & 00.4 & 'Extender cilindro C \\
\hline $\begin{array}{l}\text { SET } \\
\text { JMP TO } 1\end{array}$ & T1 & 'TIMER 1 \\
\hline
\end{tabular}

En la Figura N. ${ }^{\circ} 5$ se muestra la apariencia del software FST que permitirá la programación del PLC Festo. La programación se realiza en el lenguaje Statement List o también llamado Lista de instrucciones. Para descargar el programa al PLC, se debe instalar el driver de comunicacion TCP/IP e incluirlo en el proyecto. Para ello se ingresa a Driver Configuration y se asigna una dirección IP al PLC. Ver Figura N. ${ }^{\circ} 6$.

\section{Supervisión del sistema propuesto}

Una de las partes más importantes de los sistemas de automatización lo constituye el sistema de visualización de los datos, lo que se conoce como Interfase Hombre- Máquina (MMI) o Interfase Humano Máquina (HMI), también se denomina Software SCADA. El software SCADA o HMI está formado por diferentes software's o programas que corren en
Figura N. ${ }^{\circ}$ 5: Ventana del proyecto de la aplicación

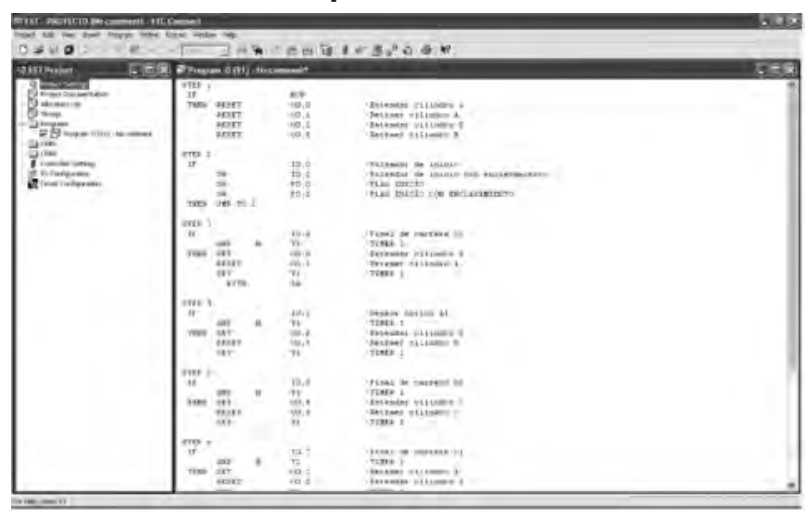

Figura N.ำ: Configuración del IP de PLC Festo

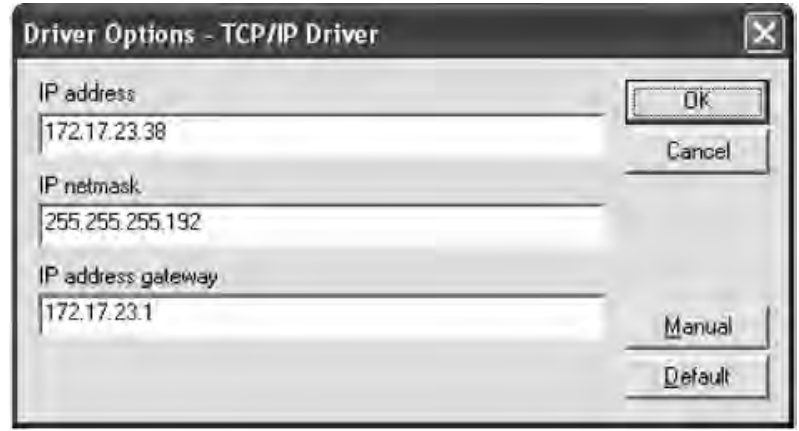

una computadora y cuyo objetivo es de visualizar todos los datos que se miden en la planta o en el campo de manera amigable, y que permita el control de la misma de manera simple y efectiva. Entonces, luego de realizar el programa de control y verificar que realmente ocurra la secuencia deseada, se trabaja con el software Intouch que es un software SCADA. En él se diseña el proceso arrastrando de su amplia librería, los elementos que se van a visualizar. El objetivo es supervisar en la pantalla del computador, lo que ocurre en el proceso y además en tiempo real. La Figura N.$^{\circ} 7$ muestra el diseño del sistema propuesto en el software Intouch.

Figura N. ${ }^{\circ}$ : Diseño del sistema propuesto en el software Intouch

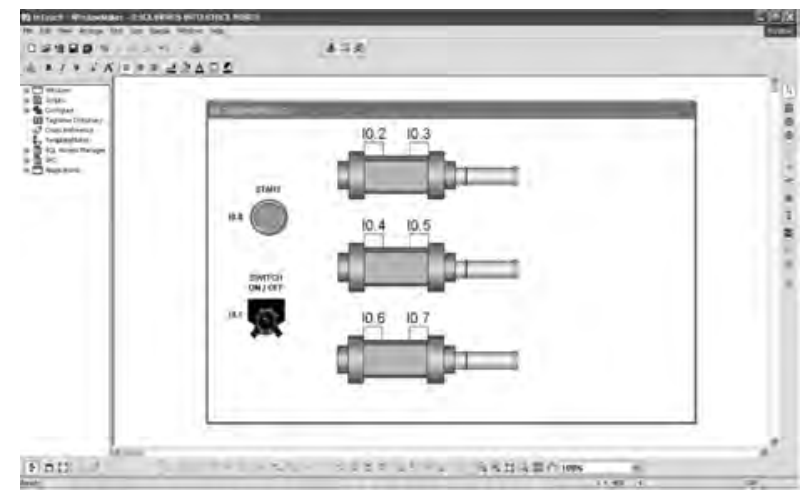

FUENTE: Elaboración propia. 
En esta etapa es indispensable la utilización del programa de comunicación IPC Data Server, el cual permite realizar la comunicación DDE entre el PC y el PLC. Ver figura 8. Este programa envía y recibe datos de los PLC's que tengan instalado el driver TCP/IP (IPC's). También se puede comunicar con PLC's que tengan solo conexión RS232 (FPC's). Este también actúa como un servidor DDE (Dinamic Data Exchange), que provee una interfase con los programas clientes DDE, en este caso el InTouch. Se requiere configurar la comunicación hacia el PLC, en nuestro caso será a través de un protocolo de red TCP/IP.

Figura N. ${ }^{\circ}$ 8: Vista del software IPC Data Server

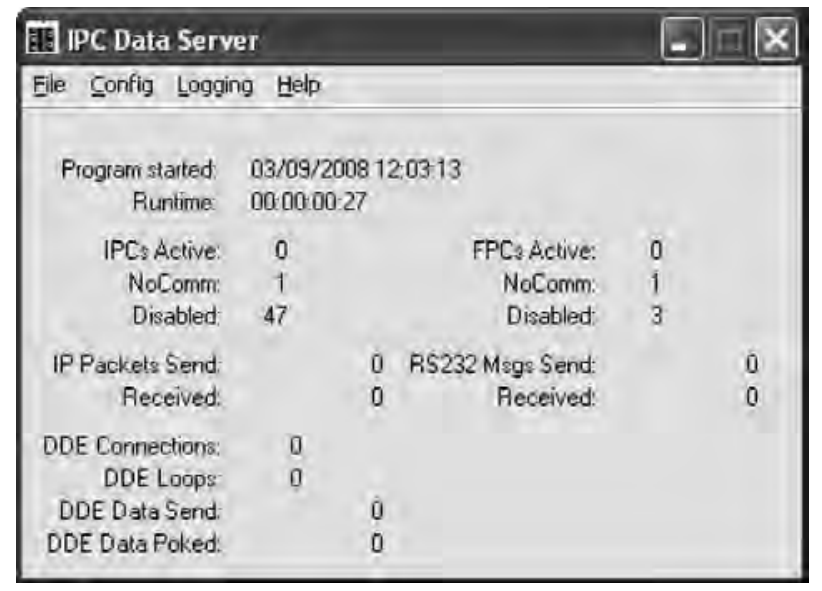

En la Figura N.$^{\circ} 9$ se muestra la ventana de configuración del protocolo TCP/IP, en ella se tiene que asignar una dirección IP al PLC, para que se pueda comunicar con este dispositivo.

Luego de configurar el protocolo de comunicación del PLC, se ingresa a la ventana Access name del Intouch para enlazar datos vía DDE de otras aplicaciones Windows hacia InTouch. Aquí es importante el nombre de "acceso" que se asigne, dado que ese nombre se utiliza más adelante en otra ventana de configuración. La Figura N.$^{\circ} 10$ muestra la ventana del Access Name.

También se requiere configurar en el Intouch todas las señales que se emplearan en la supervisión. Se debe tener en cuenta que cada señal se configurara tipo I/O Discrete y adicionalmente mediante el botón Access Name, se selecciona el "acceso" creado anteriormente. En la Figura N. ${ }^{\circ} 11$ se muestra la correcta configuracion de las señales $1 / O$ que se realiza en el Intouch.
Figura N. ${ }^{\circ}$ 9: Configuración del IPC Data Server

\begin{tabular}{|c|c|c|}
\hline \multicolumn{2}{|l|}{ Configuration for IPC 1} & $\mathrm{x}$ \\
\hline \multicolumn{3}{|l|}{$\sqrt{\nabla}$ Enabled } \\
\hline IPAddress $\longdiv { 1 7 2 . 1 7 . 2 3 . 3 8 }$ & 601 & \\
\hline$\sqrt{\square}$ Get Input Words & 『v Get Flag Words liom. & \\
\hline$\sqrt{\checkmark}$ Get Output Words & I Get Flag Words fram. & \\
\hline TV Get Registers & $\Gamma$ Get Flag Words hom: & \\
\hline \multicolumn{2}{|c|}{ Iv Get Timet Preselects $\Gamma$ Get Flag Words from: } & \\
\hline Turbo $\mp$ Update & speed & \\
\hline \multicolumn{3}{|c|}{ Updatetime for IPC information: 500 [msec] } \\
\hline No Datafile & $\nabla$ & \\
\hline Trigger Flag Word: 0 & Number of FWs: 1 & \\
\hline 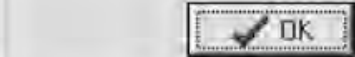 & $X$ Cancel & \\
\hline
\end{tabular}

Figura N. ${ }^{\circ} 10$ : Configuración del Access Name

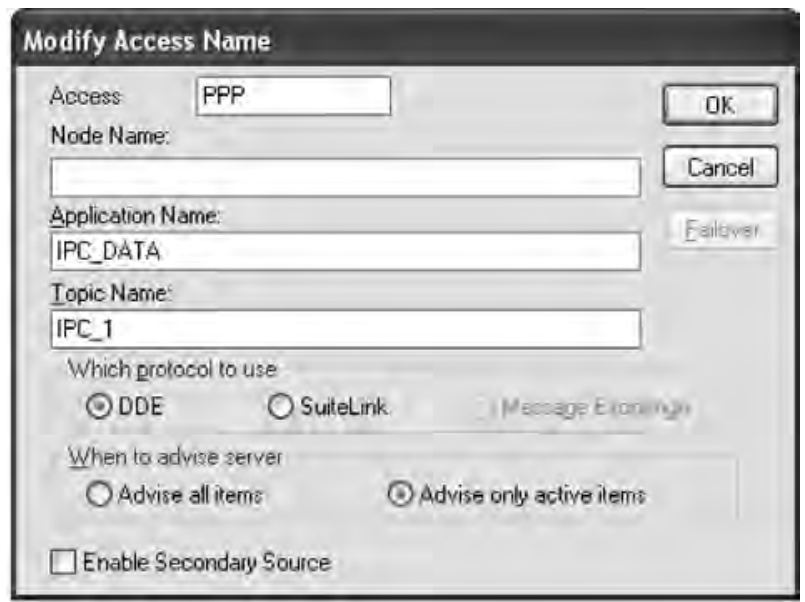

Finalmente, desde el Intouch, se ingresa a Runtime y se logrará supervisar en tiempo real la secuencia Electro-neumática. La Figura N ${ }^{\circ} 12$ muestra la vista del WindowViewer del Intouch, desde donde se puede controlar el proceso con el botón Start o el Switch ON/OFF. Si se desea que la secuencia se repita un solo ciclo, se presiona el botón Start y si se quiere que la secuencia sea continua, se presiona el Switch. La supervisión se vera en tiempo real en la pantalla del computador. 
Figura N. ${ }^{\circ}$ 11: Configuración de las señales $\mathrm{I} / \mathrm{O}$ en el Intouch



\section{CONCLUSION}

1. En el diseño del modelo electro-neumático se emplea el software FluidSim, el cual también permite simular su funcionamiento.

2. montaje de la aplicación real se realiza tomando en cuenta cada una de las señales configuradas en el software FluidSim.

3. La programación de la secuencia para la aplicación real se realiza en el software FST. Dicho programa debe ser descargado al PLC.

4. Existen parámetros como la dirección IP y otros que se deben configurar para que se realice la correcta comunicación entre el proceso electroneumático y el software Intouch.

5. Se logra el control y supervisión en tiempo real del sistema electro-neumático a través de una pantalla previamente diseñada en el Intouch.
Figura N..$^{\circ}$ 12: Vista del WindowViewer de Intouch

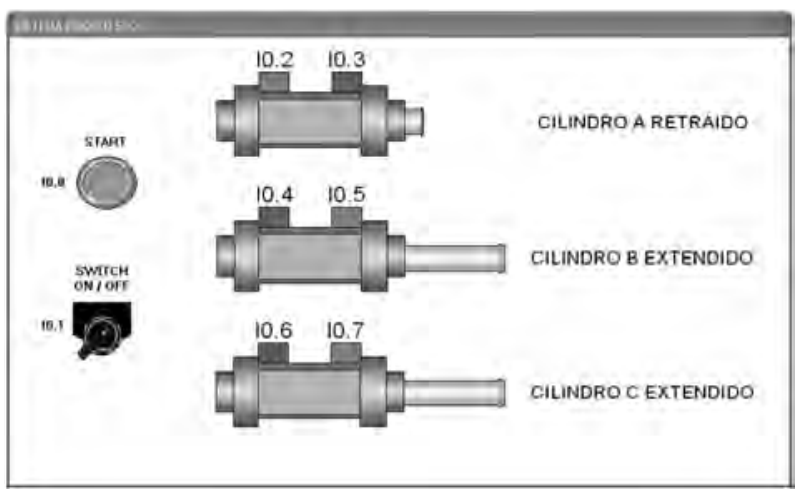

FUENTE: Elaboración propia.

\section{REFERENCIAS BIBLIOGRÁFICAS}

1. Bolton, W. (2006). Mecatrónica.

2. García Moreno, Emilio (2001). Automatización de Proceso Industriales.

3. Piedrafrita Moreno, Ramón (2001). Ingeniería de la Automatización Industrial.

4. FST User's Guide. Festo.

5. InTouch Users Guide. Invensys Systems. Wonderware. 